\title{
Portable 5.8ghz FPV system with Smartaudio
}

\author{
Idaniel Ilai David ${ }^{1}$, Jeremy Dalina ${ }^{2}$, Rick Donald Manzon ${ }^{3}$, Ryan John De Lara, Michael John Villar ${ }^{5}$ \\ ${ }^{1,2}$ Holy Angel University Angeles City, Pampanga 2009 \\ ${ }^{3,4,5}$ Nueva Ecija University of Science and Technology Gen. Tinio Street, Cabanatuan City 3100
}

\begin{abstract}
The Portable FPV System with Smartaudio is a method used simply to control a radio-controlled vehicle specifically those that use Unmanned Aerial Systems (UAS) or other type of Unmanned Aerial Vehicles (UAV) in the driver or Pilot's view point. Incorporated to the system is a special feature called Smartaudio. Smartaudio is a type of VTX telemetry protocol in which it is a combination of an audio signal and a digital control signal. The two said signals are combined together to one simple audio signal. This signal can be used to control the VTX or Video Transmitter. And it is a simple serial signal. This basically allows the Pilot of the UAV to change settings for video transmitter via the On Screen Display (OSD) menu. So having Smartaudio feature makes things like switching VTX channels, power levels less difficult for Pilots, with no need to push switches or obscure push button menus. When pilots switched over to this system that supports Smartaudio it is seen just how quickly it can change multiple settings using OSD, giving more freedom and accessibility.
\end{abstract}

KEYWORDS: FPV System, Smartaudio, VTX Telemetry, VTX Channels, UAV, UAS

\section{INTRODUCTION}

The new sport of drone building sees small but very fast robots fly around a circuit littered with obstacles. Unlike motorsports we are familiar with, the course of a drone flying can be three-dimensional, with obstacles they need to fly around, under, over and even through. Basically the UAV pilot stay on the ground but their view was as if they were inside the aircraft. This technique is known as first-personview, or FPV. The main elements of a drone set-up are the drone itself, an on-board video camera, a decent video transmitter, a pair of immersive video goggles and a set of remote controls. For an FPV setup, all you need is a video transmitter, micro camera and a goggles or FPV monitor for a receiver. But the main concern of every pilot every time they fly FPV with or without other pilots is what we call IMD (Intermodulation Distortion). Configuring the best frequencies to operate FPV system on can be a challenging prospect from time to time. There are things outside of the control may interfere with the signal, such as WiFi signals, mobile phone towers, and even other UAVs/UAS. Frequency contamination is a problem everyone faces in today's wireless world. Every VTX (Video Transmitters) on the market are built to have different channels/frequencies within the range of $5.8 \mathrm{ghz}$ spectrum because of IMD. Intermodulation Distortion happens when there are two signals that are interfering with each other. This has bad effect on the users signal. In most cases, the result is the same; it creates interference in the signal which leads to static or loss of signal in the goggles. To solve this, every pilot flying at the same time must change their channels away from each other to avoid IMD. This is quite a hassle for most pilots because to do this, either you insert your Flight controller to your computer and manually changes it through Betaflight application or creates and modifies obscure push switches to control VTX telemetry. That's why VTX telemetry control using smartaudio solves the problem of accessibility and freedom of control over these settings. Smartaudio is a combined out audio signal and a digital control signal. Audio Signal is provided from a microphone. This digital signal can be used to remotely control the Video Transmitter of the system. Adding a smartaudio feature to the system helps drone pilots control the channel the transmitter is transmitting and making the setup portable will enable pilots, engineers and drone enthusiasts build this setup to any UAS/UAVs.

\section{OBJECTIVE OF THE STUDY}

\section{A. General Objective}

The study is aimed to design a system and apply existing technology into the system to develop a device capable of VTX Telemetry protocol that can be used by any UAS or UAVs.

\section{B. Specific Objectives}

Specifically, the study is aimed to:

- Design a system capable of controlling VTX channels, power levels using OSD menu.

- Develop the system using Holybro Kakute F7 Flight Controller and Holybro Atlatl HV V1 5.8ghz 40Ch 25/200/400/600mW FPV Transmitter capable of Smartaudio.

- Configure the system using Betaflight configurator. 
- Test and Evaluate the system based on reliability and compare the time to configure the system from UAV with and without the Smartaudio feature.

\section{RESEARCH METHODOLOGY}

\section{A. System Diagram}

Figure 1 shows the simplistic diagram to help explain the system wiring. The key for a VTX telemetry control is that an extra wire is connected to a spare UART TX port on the flight controller. This UART port is what sends the information to the VTX to change the settings.

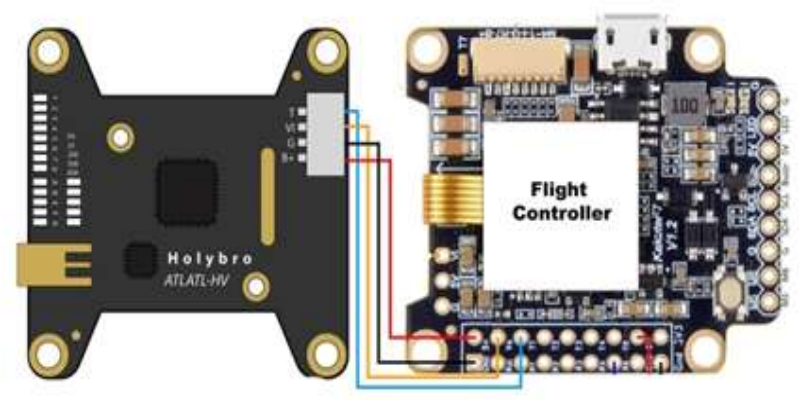

Figure 1. Wiring Diagram of Portable 5.8ghz FPV System with Smartaudio

The black wire represents the ground wire; red wire for the battery voltage input; yellow wire for video output transmitted by the VTX; blue wire will be the one connected to the UART port of the Flight controller to send signals to the Video transmitter for control and telemetry output.

Figure 2 shows the Unmanned Aerial System with Portable FPV System with SmartAudio. This includes the Electronic Speed Controller to distribute power coming from the $4 \mathrm{~s}-6 \mathrm{~s}$ lithium polymer battery distributing it to the Flight Controller and translates pilot controls into instructions sent to the motors.

\section{B. Configuration and Setup}

This contains the process in configuring the system using Betaflight configurator to enable the smartaudio feature in the system and the equipment that will be used to control the system for testing and evaluation.

Betaflight Configurator: Within Betaflight configurator under the Ports tab telemetry VTX is enabled on the corresponding UART port that is soldered in the VTX to the Flight controller. The diagram above (on the Kakute F7 Flight Controller) it shows the connection between VTX to UART 6. In the peripherals column, for the UART 6 port, either TBS Smartaudio or IRC Tramp in the drop-down is selected. VTX Table is set for the channels and power levels the VTX will produce. This will be the frequencies and power levels the smartaudio feature can navigate. Once finished, saving is essential for the reboot of the Flight.

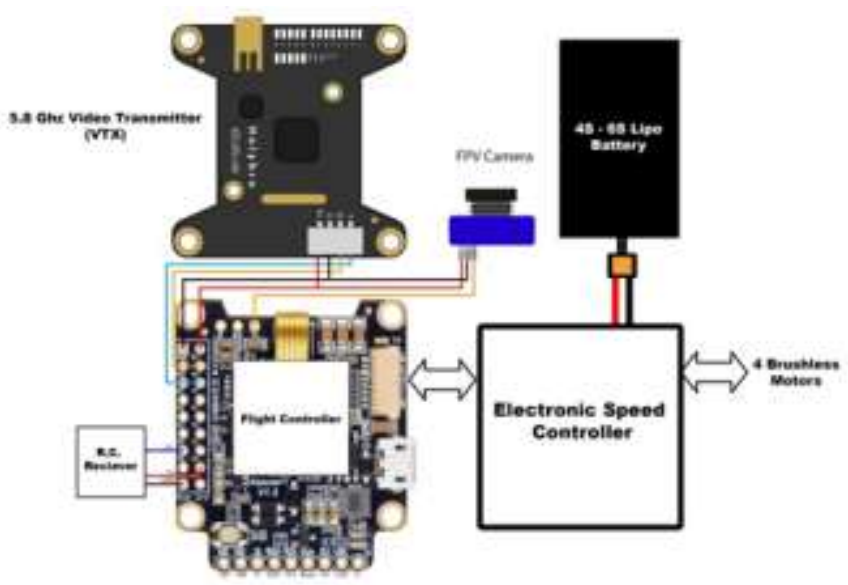

Figure 2. UAS with Portable FPV System with SmartAudio

Controller and to save all setup. After doing that and all connected correctly, the sytem will be able to access and change the VTX settings via the betaflight OSD through the controller.

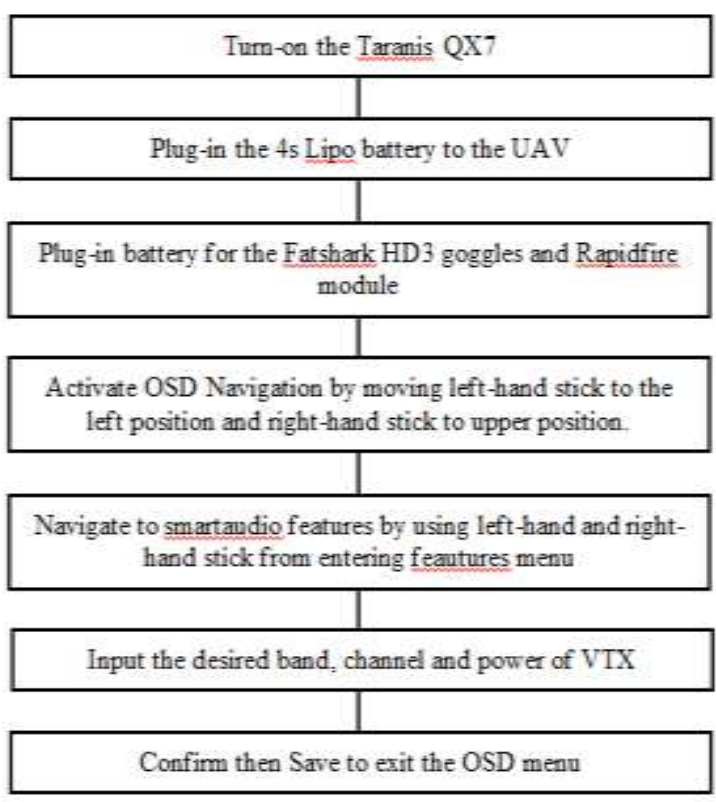

Figure 3. Smartaudio navigation flowchart

Equipment: For an FPV setup, all you need is a video transmitter, micro camera and goggles or FPV monitor for a receiver. Camera and video transmitter is already explained and shown in Figures 1 and 2. For the goggles and receiver, Fatshark HD3 and Immersion RC Rapidfire modules were used for testing. The Rapidfire module has features that will be used for testing to detect the $5.8 \mathrm{ghz}$ signal and match it from the channel the VTX is transmitting. A combination of Right Hand Circular Polarized patch and clover leaf antennas is also used in this setup. The Taranis QX7 is used as remote controller for both the UAV and On Screen Display navigation during testing the functionality of the System. Figure 3 shows the process of accessing and navigating the OSD menu to change the desired channel and power output of the $5.8 \mathrm{ghz}$ FPV system. 


\section{System Layout}

Figure 4 presents the actual project layout. The system used 5 inch carbon fiber Armattan frame to hold the system, 3 blade propellers for the 4 brushless motors and a mushroom RHCP antenna for optimum 5.8ghz signal output

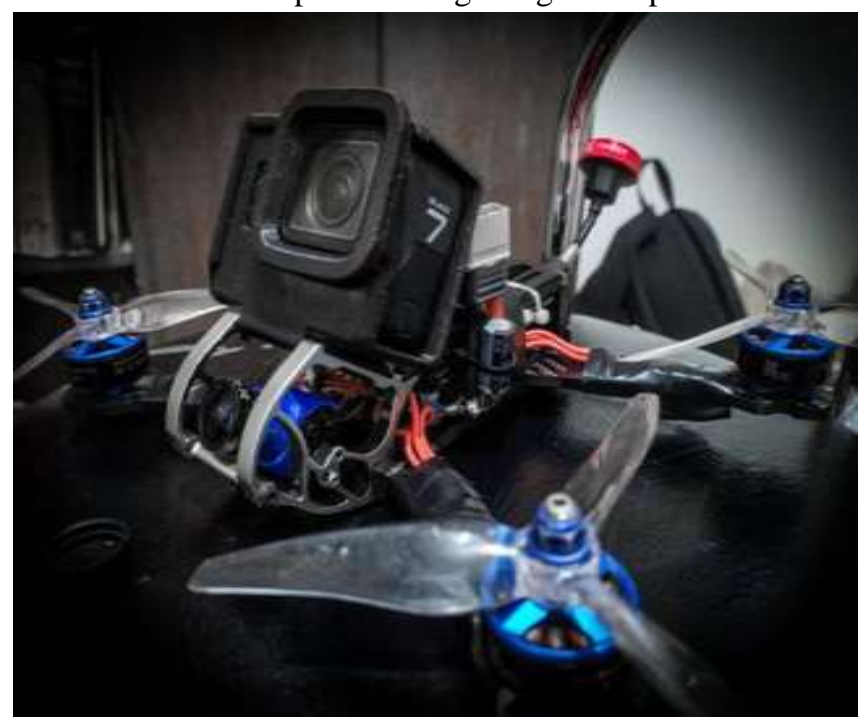

Figure 4. UAV with Portable 5.8ghz FPV system with Smartaudio

\section{IV.RESULTS AND DISCUSSIONS}

\section{A. VTX frequency channel accuracy test}

Table I-IV presents the expected frequency channel and the actual frequency channel from 4 different power modes that the transmitter is capable using also the system and the Smartaudio feature. The data gathered was proven to give $99.9 \%$ accuracy in terms of its frequency channel output.

Table I. VTX at $25 \mathrm{~mW}$ power output

\begin{tabular}{|l|l|l|l|}
\hline \multirow{2}{*}{ Trials } & $\begin{array}{l}\text { Expected } \\
\text { Frequency }\end{array}$ & $\begin{array}{l}\text { Actual } \\
\text { Frequency }\end{array}$ & \multirow{2}{*}{$\begin{array}{l}\text { Percentage } \\
\text { Error }\end{array}$} \\
\cline { 2 - 4 } & OSD & Rapidfire & \% \\
\cline { 2 - 4 } & ghz & ghz & $0.0177 \%$ \\
\hline 1 & 5.658 & 5.657 & $0.0176 \%$ \\
\hline 2 & 5.695 & 5.696 & $0.0000 \%$ \\
\hline 3 & 5.732 & 5.732 & $0.0173 \%$ \\
\hline 4 & 5.769 & 5.770 & $0.0172 \%$ \\
\hline 5 & 5.806 & 5.805 & $0.0342 \%$ \\
\hline 6 & 5.843 & 5.841 & $0.0170 \%$ \\
\hline 7 & 5.880 & 5.881 & $0.0169 \%$ \\
\hline 8 & 5.917 & 5.916 & $0.0172 \%$ \\
\hline Average Percentage Error & $99.9828 \%$ \\
\hline \multicolumn{2}{|l|}{ Reliability Test } &
\end{tabular}

Table II. VTX at $200 \mathrm{~mW}$ power output

\begin{tabular}{|l|l|l|l|}
\hline \multirow{4}{*}{ Trials } & $\begin{array}{l}\text { Expected } \\
\text { Frequency }\end{array}$ & $\begin{array}{l}\text { Actual } \\
\text { Frequency }\end{array}$ & \multirow{2}{*}{$\begin{array}{l}\text { Percentage } \\
\text { Error }\end{array}$} \\
\cline { 2 - 4 } & OSD & Rapidfire & \% \\
\cline { 2 - 4 } & ghz & ghz & $0.0884 \%$ \\
\hline 1 & 5.658 & 5.653 & $0.0176 \%$ \\
\hline 2 & 5.695 & 5.694 & \multicolumn{2}{|c}{} \\
\hline
\end{tabular}

\begin{tabular}{|l|l|l|l|}
\cline { 2 - 4 } 3 & 5.732 & 5.731 & $0.0174 \%$ \\
\hline 4 & 5.769 & 5.769 & $0.0000 \%$ \\
\hline 5 & 5.806 & 5.807 & $0.0172 \%$ \\
\hline 6 & 5.843 & 5.842 & $0.0171 \%$ \\
\hline 7 & 5.880 & 5.882 & $0.0340 \%$ \\
\hline 8 & 5.917 & 5.917 & $0.0000 \%$ \\
\hline \multicolumn{2}{|l|}{ Average Percentage Error } & $0.0240 \%$ \\
\hline \multicolumn{2}{|l}{ Reliability Test } & $99.9760 \%$ \\
\hline
\end{tabular}

Table III. VTX at $400 \mathrm{~mW}$ power output

\begin{tabular}{|l|l|l|l|}
\hline \multirow{3}{*}{ Trials } & $\begin{array}{l}\text { Expected } \\
\text { Frequency }\end{array}$ & $\begin{array}{l}\text { Actual } \\
\text { Frequency }\end{array}$ & \multirow{2}{*}{$\begin{array}{l}\text { Percentage } \\
\text { Error }\end{array}$} \\
\cline { 2 - 4 } & OSD & Rapidfire & \% \\
\cline { 2 - 4 } & ghz & ghz & $0.0353 \%$ \\
\hline 1 & 5.658 & 5.656 & $0.0176 \%$ \\
\hline 2 & 5.695 & 5.694 & $0.0349 \%$ \\
\hline 3 & 5.732 & 5.734 & $0.0520 \%$ \\
\hline 4 & 5.769 & 5.766 & $0.0344 \%$ \\
\hline 5 & 5.806 & 5.808 & $0.0171 \%$ \\
\hline 6 & 5.843 & 5.842 & $0.0170 \%$ \\
\hline 7 & 5.880 & 5.881 & $0.0169 \%$ \\
\hline 8 & 5.917 & 5.918 & $0.0282 \%$ \\
\hline Average Percentage Error & $99.9718 \%$ \\
\hline Reliability Test &
\end{tabular}

Table IV. VTX at $600 \mathrm{~mW}$ power output

\begin{tabular}{|l|l|l|l|}
\hline \multirow{3}{*}{ Trials } & $\begin{array}{l}\text { Expected } \\
\text { Frequency }\end{array}$ & $\begin{array}{l}\text { Actual } \\
\text { Frequency }\end{array}$ & \multirow{2}{*}{$\begin{array}{l}\text { Percentage } \\
\text { Error }\end{array}$} \\
\cline { 2 - 4 } & OSD & Rapidfire & \% \\
\cline { 2 - 4 } & ghz & ghz & $0.0000 \%$ \\
\hline 1 & 5.658 & 5.658 & $0.0176 \%$ \\
\hline 2 & 5.695 & 5.694 & $0.0000 \%$ \\
\hline 3 & 5.732 & 5.732 & $0.0000 \%$ \\
\hline 4 & 5.769 & 5.769 & $0.0172 \%$ \\
\hline 5 & 5.806 & 5.805 & $0.0171 \%$ \\
\hline 6 & 5.843 & 5.842 & $0.0000 \%$ \\
\hline 7 & 5.880 & 5.880 & $0.0169 \%$ \\
\hline 8 & 5.917 & 5.918 & $0.0086 \%$ \\
\hline Average Percentage Error & $99.9914 \%$ \\
\hline \multicolumn{2}{|l|}{ Reliability Test } &
\end{tabular}

\section{B. Comparison of UAS with and without Smartaudio}

Table V presents the time of setup when a power mode of the VTX and a frequency channel has been configured from an FPV system with smartaudio feature versus FPV system without smartaudio feature. Configuring power modes and frequency channels without smartaudio will be the old conventional way. The data gathered was proven to save average of 7.5 minutes time in setup configuration. 
Table V. VTX at 200mW power output

\begin{tabular}{|l|l|l|l|}
\hline \multirow{2}{*}{ Trials } & $\begin{array}{l}\text { With } \\
\text { Smartaudio }\end{array}$ & Conventional & \multirow{1}{*}{ Difference } \\
\cline { 2 - 3 } & Seconds & Seconds & 437 \\
\hline 1 & 451 & 14 & 449 \\
\hline 2 & 461 & 12 & 442 \\
\hline 3 & 457 & 15 & 439 \\
\hline 4 & 455 & 16 & 449 \\
\hline 5 & 462 & 13 & 460 \\
\hline 6 & 474 & 14 & 449 \\
\hline 7 & 462 & 13 & 461 \\
\hline 8 & 476 & 15 & 469 \\
\hline 9 & 489 & 20 & 438 \\
\hline 10 & 455 & 17 & 449.3 \\
\hline Ave: & 464.2 & 14.9 & 7.488333333 \\
\hline Average Saved time in Minutes & \\
\hline
\end{tabular}

\section{CONCLUSIONS}

FPV system with smart audio basically allows the Pilot of the UAV to change settings for video transmitter via the On Screen Display (OSD) menu. So having Smartaudio feature makes things like switching VTX channels, power levels less difficult and saves time for Pilots, with no need to push switches, obscure push button menus or connecting youre UAV to the computer to configure the setup like the conventional way. When pilots switched over to this system that supports smartaudio it is proven just how accurately and how quickly it can change multiple settings using OSD, giving more freedom and accessibility.

\section{ACKNOWLEDGMENT}

This paper and the research behind it would not have been possible without the exceptional support of our supervisors, Dr. Rick Manzon, Dr. Ryan De Lara and Dr. Michael Villar. Their enthusiasm, knowledge and exacting attention to detail have been an inspiration and kept our work up until the final draft of this paper. I would like to also acknowledge the General Manager of Edge-Systems Engineering Services, Engr. Edzel Galzuerra for utilizing the system in its projects particularly in the survey of Renewable Energy sites.

\section{REFERENCES}

1. Corrigan, Fintan. (2020, March 15). 10 Best FPV Drone Goggles For A Terrific Flying Experience. Dronezon.

https://www.dronezon.com/drone-reviews/fpvgoggles-for-drones-to-experience-the-thrill-offlying/

2. Shutts, Ian. (2021, April 21). DJI Air 2S Review of Features and Specs. dronezon. https://www.dronezon.com/drone-reviews/dji-air2s-review-of-features-and-specs/
3. Team Blacksheep. (2018, February 3). TBS SmartAudio, what it is and how it works. Team BlackSheep.

https://team-blacksheep.freshdesk.com/suppor t/solutions/articles/4000100084-tbs-smartaudiowhat-it-is-and-how-it-works

4. Ro, Lawrence. (2019, August 15). Diagnosing and Fixing Bad FPV Video. getfpv.

https://www.getfpv.com/learn/fpv-diy-repairs-andmods/diagnosing-and-fixing-bad-fpv-

video/?fbclid=IwAR03w4zPDalnAfWPb7eTnCMc IOq111OGfY2Rh6xfLF9AHIJ0vuL2rnXmYD4\#: :t ext=The $\% 20$ most $\% 20$ common $\% 20$ causes $\% 20$ for, mechanical\%20disruptions $\% 20$ in $\% 20$ the $\% 20$ quad. \&text $=$ White $\% 20$ line $\% 20$ static $\% 20$ is $\% 20$ electrical ,camera\%20and\%2For\%20video\%20transmitter

5. Thickstun, Alexander. (2016, May 18). Fat Shark's newest FPV goggles make flying a drone feel more like piloting a fighter jet. digitaltrends.

https://www.digitaltrends.com/cool-tech/fat-sharkdominator-v3-review-

video/?fbclid=IwAR3_z13Brk1Mi1iboxqzplXo5i8

Lj2HC8rdiX32VVByzAyjU_U_S35T3qr0

6. ALEX. (2017, September 4). What is VTX telemetry and why should you care? (SmartAudio and Tramp). Dronetrest.

https://blog.dronetrest.com/smart-audio-vtx/

7. Norman, A. (2015). Immersion RC Rapidfire Review. Drone Nodes.

https://dronenodes.com/immersion-rc-rapidfirereview/?fbclid=IwAR0Zsnwe-ojb-iLy-

VISbr7WsHfcVNs8H9KZKLEZVbd7D65FeRAY5 SNVvuc

8. Liang, Oscar. (2019, May 19). REVIEW: IMMERSIONRC RAPIDFIRE RECEIVER MODULE FOR FPV GOGGLES. Oscar Liang FPV Drone Tutorials and Reviews.

https://oscarliang.com/immersionrc-rapidfiremodule/

9. Montis. (2019, April 14). Guide: SmartAudio VTX control and how to set it up. Multirotor Guide. http://www.multirotorguide.com/guide/guidesmartaudio-vtx-control-and-how-to-set-it-up/

10. DJI. (2016, May 25). Inside a Drone - ESC. dji. https://www.dji.com/newsroom/news/inside-adrone-esc?fbclid=IwAR2h3uOAlOSOcFYd2Cz 1b7wcZueyH4LGHxHda9XUO13cThPHJFMFhxt Mxn4\#: :text=This\%20is\%20all\%20down\%20to,t he $\% 20$ motors $\% 20$ to $\% 20$ control\%20movement

11. RCExplorer. (2012, August 1). CLOVERLEAF ANTENNA. Flitetest.

https://www.flitetest.com/articles/Cloverleaf_Anten na?fbclid=IwAR004PfijUbwL6A-

QYJuT1jLerYqhuY 
Ni_hnUJs_ABakZIyFHYtumo9afXA\#: :text=The

$\% 20$ Cloverleaf $\% 20$ is $\% 20 \mathrm{a} \% 20$ closed,works $\% 20 \mathrm{gr}$ eat\%20as\%20an\%20antenna

12. RCExplorer. (2011, August 19). Circular polarization explained. rcexplorer.

https://rcexplorer.se/educational/2011/08/circularpolarization-explained/?fbclid=IwAR2WBpR1Ua MWnMgbcJn3U3oi6HCPv00r-VnnAOQiBEhqC pYpHCVkRt40MMM

13. John, Francis Rohan. (2020, June 21). FrSky Taranis QX7 Review with Pros \& Cons. Horusrc.

https://www.horusrc.com/en/blog/frsky-taranisqx7-review/?fbclid=IwAR3iPiu6dcNlusc5 Ni4kN9bm6F4WrAZpGTi9VtKTBjeJYR0ScJvVM 2ero9A 